\title{
Effect of Sub-maximal Exercise Stress on Cold Pressor Pain: A Gender Based Study Pokhrel BR, ${ }^{1}$ Malik SL, ${ }^{1}$ Ansari $A H,{ }^{2}$ Paudel BH, ${ }^{3}$ Sinha $R,{ }^{3}$ Sinha $\mathrm{M}^{3}$
}

Department of Physiology

${ }^{1}$ Kathmandu University School of Medical Sciences

Kavre, Nepal

${ }^{2}$ All India Institute of Medical Sciences, New Delhi, India ${ }^{3}$ B.P.Koirala Institute of Health Sciences, Dharan, Nepal

\section{Corresponding Author}

Bhola Raj Pokhrel

Department of Physiology

Kathmandu University School of Medical Sciences

Kavre, Nepal

E-Mail: bholapokhrel@gmail.com

\section{Citation}

Pokhrel BR, Malik SL, Ansari AH, Paudel BH, Sinha R, Sinha M. Effect of Sub-maximal Exercise Stress on Cold Pressor Pain: A Gender Based Study. Kathmandu Univ Med J 2013;41(1):54-59.

\section{ABSTRACT}

\section{Background}

Analgesic effect of exercise is a well established fact; however available reports are contradictory on gender differences in pain perception following exercise stress test.

\section{Objective}

The current study is prospectively designed to evaluate and compare the effect of acute bout of sub-maximal exercise stress on cold pressor pain in normal adult males and females.

\section{Method}

The experimental study design involved healthy adults $(n=41)$; females $(n=18)$ and males ( $n=23$ ) aged between 18 to 25 years and included them into four sets of experiments: SET I (Control), "resting blood pressure, radial pulse and respiratory rate were recorded after 15 minutes of complete supine relaxation. SET II (Cold Pressor Pain Test): Pain Threshold, Pain Tolerance, and Pain Duration in seconds were taken. SET III (Exercise Stress Test): Sub-maximal exercise of 70 to $75 \%$ of maximum predicted heart rate was given for 6 minutes. SET IV (Cold Pressor Pain Test immediately after Exercise Stress Test): At 0 minute of recovery again the pain parameters; Pain Threshold, Pain Tolerance, and Pain Duration in seconds were taken. SET I, SET II were performed in order on the first day and SET III and SET IV on the second day to ensure only a single Cold Pressor Pain Test is performed in each day.

\section{Result}

The data (Mean \pm SD) analysis showed significant increased in pain threshold (males: $14.36 \pm 10.6$ Vs $21.47 \pm 13$ seconds, $p<0.001$, females: $14.1 \pm 11.5$ Vs $23.81 \pm$ 20.22 seconds, $p<0.001$ ) and pain tolerance time (males: $41.3 \pm 19.31 \mathrm{Vs} 54.1 \pm 21.7$ seconds, $p<0.001$ ) in both sexes after 6 min of acute bout of sub-maximal exercise stress test with comparable age, $\mathrm{BMI}$ and baseline resting values of pain parameters and pulse rate and blood pressure. The percentage increment pain tolerance time following the exercise stress in female is higher than male (78.6 Vs 68.9\%).

\section{Conclusion}

The result suggests that pain sensation decreases immediately after a brief period of exercise challenge irrespective of gender, and the analgesic effect of the acute bout of exercise in terms of pain tolerant time is more enhanced in females than males.

\section{KEY WORDS}

Analgesia, exercise, gender, pain tolerance 


\section{INTRODUCTION}

Exercise stress has been reported to alter sympathetic activity and muscle pain perception. ${ }^{1}$ One of the neuroendocrine responses to stress includes release of endogenous opioid and non-opioid substances in the circulation, which accounts for stress induced modulation of pain observed in experimental or clinical pain models. ${ }^{2,3}$ However, there are contradicting reports regarding exercise-induced analgesia. The analgesic effect of exercise may be entirely or in part a pain test artifact. ${ }^{4}$ A work of Sternberg et al, 1992 suggests that analgesia during competition in athletes may be attributed to the associated motivational factors and not only to the related physical stress, per se. ${ }^{3}$ Similarly, Bartholmew experiment on analgesic effect of intensity and condition of exercises supports the prediction that the analgesic effect of exercise is not limited to the controlled environment of experiment, but to other naturally occurring situations as well. ${ }^{5}$ Stress of interpersonal competition may be a more significant factor than physical exertion in decreasing pain perception in case of male. On the other hand, women pain perception appears to be more affected by the physical exercise rather than the stress of competition. ${ }^{3}$

There are increasing evidences of sex differences in the experience of pain for women and men. ${ }^{6-11}$ Biological and psychological factors are both important in explaining sex and gender differences in pain experience. ${ }^{9}$ Cold pressor pain studies suggest that though pain tolerance is greater in male, the behavior of perception differs in women with different phases of menstrual cycle and thus analgesic effect of stress. ${ }^{12,13}$ Present study has observed the gender differences of analgesic effect of exercise stress taking these variations and contradiction of gender differences in consideration. To overcome methodological pitfall as found in other studies, where stress induced analgesia by pain itself was the potentially confounding factor, our experimental design had the provision to isolate the responses to pain test alone from the exercise induced modulation of pain by conducting pain test in two separate days for each subject. ${ }^{14-16}$

\section{METHODS}

Total forty-one $(n=41)$ healthy adult volunteers; twentythree males and eighteen females selected by simple random sampling technique. All of the subjects were medical students and employees of BP Koirala Institute of Health Sciences, Dharan and the study was carried out at the laboratory of Department of Physiology in the same institute. A questionnaire was used to collect the following information from each subject: personal data, age, sex, place of growing, and occupation, and all were screened by physician-acquired history. In case of the female subjects, the recordings were taken during mid-luteal (21 to 24 days) phase of their menstrual cycle to avoid any effects of cyclical changes in pain perception. Any subjects presented with signs/ symptoms of cardio-respiratory diseases or pain related or autonomic dysfunctions and under medication or symptomatic individuals with exercise stress test were excluded in the study. Signed written informed consent was taken from each participating individual which was approved my BPKIHS ethical committee.

The experiment was designed to assess the effect of exercise on the pain perception and tolerance in males and females. Four sets of experiments were carried out in temperature controlled $\left(25 \pm 2{ }^{\circ} \mathrm{C}\right)$ laboratory in each subject, irrespective of gender. SET I (Control): After measurement of Body Height and Weight which was used in BMI calculation, the vital signs such as Heart Rate, Blood pressure and Respiratory Rate were recorded after 15 minutes of complete supine relaxation. SET II (Cold Pressor Pain Test): Pain Threshold, Pain Tolerance, and Pain Duration in seconds were taken. SET III (Exercise Stress Test): Submaximal exercise of 70 to $75 \%$ of maximum predicted heart rate was given for six minutes. SET IV (Cold Pressor Pain Test immediately after Exercise Stress Test): At 0 minute of recovery again the pain parameters; Pain Threshold, Pain Tolerance, and Pain Duration in seconds were taken. SET I, SET II were performed in order on the first day and SET III and SET IV on the second day to ensure only a single Cold Pressor Pain Test is performed in each day.

This is a standard non-invasive test for acute pain complying with the ethical guidelines by the International association for the study of pain. ${ }^{17}$ The pain induced by the test is reversible and does not cause any tissue damage of the individual. This was carried out on the first day under resting condition and immediately after exercise on the second day of the experiment. Well instruction about the procedure was given before the experiment. After complete relaxation, subject was asked to immerse his/ her dominant hand in the ice water maintained at $1.5 \pm 0.5$ oC and to report sensation of pain by raising another free hand when begun and to take the hand out when it became intolerable. A maximum time of three minutes was allowed for every participant to remain the hand immersed in ice water in case the subject did not report intolerable pain, to avoid any permanent tissue damage. ${ }^{18}$ Two stop watches were simultaneously used to record Pain Threshold and Pain Tolerance Time in seconds. Pain Threshold was the time noted between the immersion of hand to the first feeling of pain and Pain Tolerance was the total time the subject kept hand immersed in ice water till the pain rises to intolerable. By subtracting Pain Threshold from the Pain Tolerance time the Pain Duration was calculated.

It was performed on the bicycle ergometer according to standard WHO protocol. ${ }^{19}$ At least two hours after the light breakfast in the morning hours between 8:00 to 12:00 the participants were advised to come. The test was conducted on a programmable bicycle ergometer (Ergoline D72475 Bitz, Germany) beginning with 25 watts of exercise load. At every interval of 2 minutes the bicycle was programmed to increase load resistance by 25 watts and was performed for 
4 to 5 steps till the subject reached 70 to $75 \%$ of maximum predicted heart rate. Heart Rate and blood pressure was continuously monitored using wrist model automatic Blood pressure and Heart Rate Meter (EW 272, Matsushita Electric Works Ltd, Japan) for up to 6 minutes after the subject reached sub-maximal level of exercise.

The significance of difference in pain perception between males and females were analyzed using non-parametric tests Kruskul-Wallis and Mann-Whitney $U$ test and to compare difference of the means between pre- and postexercise changes in CPPT within participants of the same gender Wilcoxon Signed Ranks test was used. The values were expressed as Mean and Standard Deviation and ' $p$ ' values less than 0.05 were deemed significant. For all the statistical analyses, EPI Info (2000) software was used.

\section{RESULTS}

Demographic information and BP recorded at resting stage and during exercise stress are shown in Table 1 . All the 41 participants had age range between 18 to 30 years. There were no overall sex differences in age; however, females were slightly younger than males. BMI was also found comparable between the sexes. The variation of pulse rate responses during exercise for each individual fallen between 70 to $80 \%$ of maximum predicted heart rate and the post-exercise response did not differ significantly in sexes.

Table1. Demographic data and the values recorded during resting and sub-maximal exercise stress test.

\begin{tabular}{|lcc|}
\hline & Male & Female \\
\hline Age (years) & $24.04 \pm 3.9$ & $21.22 \pm 2.6$ \\
\hline BMI (Kg/m2) & $22.28 \pm 1.7$ & $22.07 \pm 1.6$ \\
\hline During Resting & & \\
\hline SBP (mmHg) & $116.25 \pm 6.5$ & $113.34 \pm 4.9$ \\
\hline DBP (mmHg) & $69.87 \pm 7.6$ & $65.05 \pm 7.1$ \\
\hline PR (beats/ min) & $78.01 \pm 15.9$ & $80.02 \pm 13.3$ \\
\hline During Sub-Maximal Exercise (Average of recorded values) \\
\hline SBP (mmHg) & $151 \pm 20$ & $169 \pm 13$ \\
\hline DBP (mmHg) & $86 \pm 13.5$ & $91.87 \pm 11.5$ \\
\hline PR (beats/ min) & $149 \pm 14$ & $156 \pm 9.4$ \\
\hline
\end{tabular}

Following CPPT alone, we found the average pain threshold and pain tolerance time was insignificantly $(p<0.82)$ differed between males and females. But still, the pain tolerance time was found higher in males whereas pain threshold was comparable in both sexes. However, after the exercise stress test, both the pain parameters increased significantly in males and females (Table 2). During EX+CPPT, cold tolerance time was at $p<0.001$ and pain threshold at $p<0.01$ level of significant differences compared to their baseline recorded values during CPPT alone. While calculating the percentage changes from the baselines during EX+CPPT (Fig 1), we found that percentage

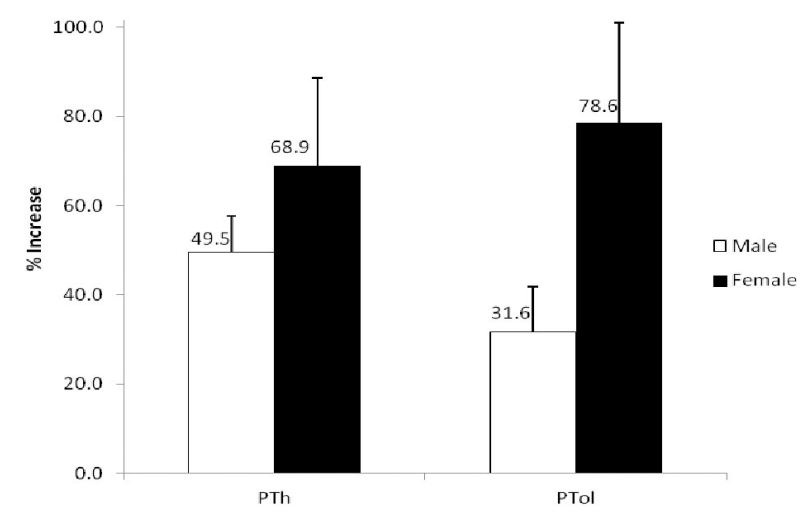

Figure 1. Percentage increase in PTh and PTol from its baseline after the exercise stress test.

Table2. Comparison of the average threshold time and pain tolerance time between CPPT and CPPT after the exercise (EX+ CPPT) in males and females.

\begin{tabular}{lllll} 
Sex & \multicolumn{2}{l}{ CPP-Threshold time } & \multicolumn{2}{l}{ CPP-Tolerance time } \\
& CPPT & EX+ CPPT & CPPT & EX+ CPPT \\
\hline Male & $14.36 \pm 10.6$ & $21.47 \pm 13^{\mathrm{a}}$ & $41.3 \pm 19.31$ & $54.1 \pm 21.7^{\mathrm{b}}$ \\
Female & $14.1 \pm 11.5$ & $23.81 \pm 20.22^{\mathrm{a}}$ & $37.44 \pm 22.2$ & $66.85 \pm 25.88^{\mathrm{b}}$
\end{tabular}

Average time points are in unit of seconds and expressed as mean \pm SD. (a) indicates $p<0.001$ for threshold time and $\left({ }^{b}\right)$ indicates $p<0.01$ for tolerance time compared to its baseline CPPT in males and females. Abbreviation used: CPP: Cold Pressor Pain. CPPT: Cold Pressor Pain Test. CPPT+EX: Cold Pressor Pain Test after the Exercise.

increment in the pain tolerance time (78.6\%) was higher than the percentage increase of pain threshold time (68.9\%) in females. In males, the percentage increments of these parameters were lower than females. The comparison of post exercise values of pain threshold and pain tolerance time showed no significant differences in pain responses between males and females. During analysis, we excluded the data of the participants who reached or exceeded the 3 minutes of cut-off limit of hand immersion duration in cold pressor pain test before or after the exercise.

\section{DISCUSSION}

The present study adds the evidences that exercise does cause alteration in pain perception basically producing the analgesic effects, irrespective of gender. Hence, the pain responses noted before the exercise stress test as moderately painful was significantly reduced after the stress test, suggesting an increased pain threshold and pain tolerance time. It can be explained with possible role of endogenous opioid system which is stimulated by physical exercise and is responsible for exerciseinduced analgesia. ${ }^{14,16}$ Our finding was consistent with the observation of Pertovaara on dental pain threshold and the release of stress hormones. ${ }^{15}$ In animal model of study, Shyu had also reported an increase in pain threshold after long lasting exercise in rats. ${ }^{20}$ In addition to that, a work of Kuoppasalmi reported an increment in discharges in proprioceptive and other muscle afferents during exercise which possibly activate inhibitory mechanisms at spinal or 
supraspinal levels in a way suggested by the gate control theory of pain. ${ }^{21}$

While general mechanisms of stress-induced antinociception may be invoked to explain exercise analgesia, for example the descending inhibitory pathways of the spinal cord dorsolateral funiculus, there have been few attempts to specify exactly how exercise interacts with those systems. ${ }^{22}$ Thoren et al presented a credible model of exercise specific analgesia. ${ }^{23}$ The key element in their model involves afferent activity from A-delta fibers located in large muscle groups. Electrical stimulation of such fibers in animals has been shown to increase central opioid activity and to produce naloxone-reversible analgesia. Exercise of these large muscle groups has also been shown to produce activity in these afferent fibers.

In our study, pre-exercise resting values of pain threshold was observed similar in both male and female groups indicating that pain sensitivity is similar. This is supported by Casey $\mathrm{KL}$ report stating that pain threshold is same for everyone: that is, people perceive it at the same stimulus intensity. ${ }^{24}$ Similarly, study done by Hellstrom and Lundberg examined the pain thresholds and had found no significant differences between males and females. ${ }^{12}$ The pain tolerance time, the other parameter of pain was found higher in males than females but significance of difference was not seen. The finding of lesser pain tolerance has been consistent through the research done with experimentally induced pain..$^{25,17,19,26}$

Within gender, pain tolerance time seems to be further influenced by both psychological and cultural backgrounds and physiological factors such as athletic participation. ${ }^{25,27}$ A study has found that Eastern Cultures are more likely to have a higher pain tolerance than Western Cultures. ${ }^{28}$ The groups with different cultural philosophies and spiritualities that could, in part, explain the differences in pain tolerance. ${ }^{25}$ Based on these it can be suggested that a strong psychological component prevails in the expression of pain tolerance. Regardless of biological differences, that one can show a significant higher level of pain tolerance based solely on psychological processes instilled by a person's culture. If this is true between cultures then it could also explain for gender differences. The belief throughout most cultures is that, it is more accepted for women to express their pain while men are supposed to be strong and hide their pain. In addition, from the biological perspective lower levels of pain tolerance in females also may be related with different neuronal pain path for pain perception, which was found to be present in female rodents. ${ }^{29}$ Some of the studies also provided evidences that pain sensation evoked by the cold pressor test was increased during the late luteal phase in normal menstruating women which was related to the serum concentration of progesterone or on changes in the S-progesterone level. ${ }^{30,31}$ However, in our study its influence on gender differences of pain perception was not noticed even after recording at mid-luteal phase of menstrual cycle.
The modification of pain perception can be produced by the physical exercise which reduces sensitivity to noxious stimuli, so called 'Exercise Induced Analgesia or (EIA)' and since exogenous and endogenous opioids produce analgesia, it is reasonable to expect that exercise would act as a trigger for endogenous analgesia expression in human. ${ }^{14,32}$ Our report showed that exercise increased the magnitude of pain threshold and pain tolerance significantly when EX+ CPPT was done as compared to CPPT alone, in both the genders. When CPPT was performed immediately after exercise, $B P, H R$ and RR were already high just before doing CPPT. The EIA might be the cause of increase pain threshold and pain tolerance in all subjects. In a study by Cook and Pertovara, it was shown that exercise stress altered the sympathetic activity and pain perception. ${ }^{1,5,16}$

Because of differential analgesic hormonal responses to exercise in male and female the pain sensitivity was more decreased in females particularly pain tolerance time. ${ }^{33}$ EIA have been found to be relatively enhanced in females as estrogen appears to be responsible for the impact of estrous cycle on opioid analgesia. This suggests that ovarian hormones may modulate the impact of stressors on endogenous pain inhibition and other stress-responsive systems. These unequal responsiveness is also supported by al' Absi M while observing the responses to naltrexone, women exhibited reduced blood pressure and increased pain tolerance after CPPT. ${ }^{34}$

Our study design differed from earlier studies for EIA, where most of the research performed the pain test after few minutes gap after exercise challenge. ${ }^{14-16}$ CPPT was performed immediately after exercise that might also be the cause of enhanced effect than the other studies. Another difference was in the intensity of exercise. In our exercise protocol, the intensity of exercise was at the level of $70 \%$ of maximum predicted heart rate, which might have been sufficient for the activation of EIA. Similar type of response was also found in the study of Petovaara15 where exercising at a sub-maximal aerobic workload, $70 \%$ of maximal work capacity, was enough to produce dental pain threshold elevation. However in general it is very difficult to determine the intensity and duration of exercise, which might produce analgesia, as the experimental protocols are different in different studies. It is clear that pain parameters vary greatly among individuals, comparison of results with the different subjects acting as control and experimental becomes erratic. ${ }^{18}$ But in our study same subject acted as their own control as well as experimental, therefore reducing the chances of inter-group variation in pain perception.

Although the effect of exercise is significant on pain in both male and female, the effect is higher in female than male. Initially pain threshold was comparable in both the groups but after the exercise value increased more in female than male. However, the gender differences in the pain parameters were not statistically significant (Table 2). Also in the study by Kowalzkyk et al in which the number 
of participants was almost comparable to the present study (20 men and 21 normally menstruating women, vs. 23 and 18 in the present study), no statistically significant differences between men and women were observed. ${ }^{35}$ Previous studies specifically designed to investigate gender differences, in which the number of participants has been of the order of 200 have demonstrated significant sex differences in response to the cold pressor test. ${ }^{36-38}$ Hence, this seems to be more likely explained by sample size, which is the limitation of our study.

\section{CONCLUSION}

The present study design overcomes the methodological pitfalls which leads to adaptation of cold pressor test as

\section{REFERENCES}

1. Cook DB, Connor PJ, Ray CA. Muscle pain perception and sympathetic nerve activity to exercise opoid modulation. Am J Physiol Regul Integr Comp Physiol 2000; 279:1565-73.

2. Sen P, Khanna N, Ray A. Histaminergic mechanisms in experimental convulsions. Indian J Exp Biol 1991; 29: 375-8.

3. Sternberg WF, Bailin D, Grant M, Gracely RH. Competition alters the perception of noxious stimuli in male and female athletes. Pain 1998; 76:231-8.

4. Padawer WJ, Levine FM. Exercise-induced analgesia: fact or artifact? Pain 1992; 48(2): 131-5.

5. Bartholomew JB, Lewis BP, Linder DE, Cook DB. Post-exercise analgesia: replication and extension. J Sports Sci 1996; 14(4): 329-34.

6. Unruh AM. Gender variations in clinical pain experience. Pain 1996; 65(2-3): 123-67.

7. Berkley KJ. Sex differences in pain. Behavioral and Brain Sciences 1997; 20 (03): 371-380.

8. LeResche L. Gender considerations. In: Crombie IK, Croft PR, Linton SJ, LeResche R, Von Korff M. Epidemiology of Pain IASP 1999.

9. Unruh AM. Why can't be a woman more like a man? Behavioral and Brain Sciences 1997; 20:467-8.

10. Unruh AM, Ritchie JA, Merskey H. Does gender affect appropriate of pain and pain coping strategies? Clinical Journal of pain 1999; 15:3140.

11. Unruh AM, Campbell MA. Gender variation in children's pain experience. In: McGrath PJ, Finley GA. Chronic and recurrent pain in children and adolescents. Progress in pain Research and Management 1999, 13: 199-241.

12. Hellström B, Lundberg U. Pain perception to the cold pressor test during the menstrual cycle in relation to estrogen levels and a comparison with men. Integr Physiol Behav Sci 2000; 35(2): 132-41.

13. Al'Absi M, Petersen KL and Wittmers LE. Adrenocortical and hemodynamic predictors of pain perception in men and women. Pain 2002; 96(1-2): 197-204.

14. Janal MN, Glusman M, Kuhl JP and Clark WC. Are runners stoical? An examination of apn sensitivity in habitual runners and normally active controls. Pain 1994; 58: 109-16.

15. Pertovaara A, Huopaniemi T, Virtanen A, Johansson G. The influence of exercise on dental pain thresholds and the release of stress hormones. Physiol and Behav 1984; 33: 923-26.

16. Kemppainen P, Pertovaara A, Huopaniem T, Johansson G, Karonen SL. Modification of dental pain and cutaneous thermal sensitivity by physical exercise in man. Brain Research 1985; 360: 30-40. employed in other studies and avoid intergroup variation by taking same subject as its control for pain perception and with this background it provides the evidence that pain sensation evoked by the cold pressor test, a tonic thermal stimulus, is decreased immediately after a brief period of exercise challenge irrespective of gender, and the analgesic effect of the acute bout of exercise in terms of pain tolerant time is more enhanced in females than males, which possibly dependent on the serum concentration of progesterone or on changes in the S-progesterone level in the mid-leuteal phase of menstrual cycle of female. These data, hence, provides information on gender differences in experimentally induced pain and analgesic effect of exercise based on novel experimental design.

17. Walsh NE, Schoenfeld L, Ramamurthy S, Hoffman J. Normative model for cold pressor test. Am J Phys Med Rehab 1989; 68: 6-11.

18. Tandon OP, Himani A, Singh $\mathrm{H}$. Pulmonary responses during cold induced acute pain. Indian J Physiol pharmacol 1997; 41: 16-22.

19. Westcott TB, Huesz L, Boswell D, Herold P. Several variables of importance in the use of the cold pressor as a noxious stimulus in behavioral research. Percept Motor Skills 1977; 44:401-2.

20. Shyu BC, Andersson SA, Thoren P. Endorphin mediated increase in pain threshold induced by long lasting exercise in rats. Life Sciences 1982; 30: 833-40.

21. Rashed HM, Leventhal G, Madu EC, Reddy R, Cardiso S. Reproducibility of exercise-induced modulation of cardiovascular responses to cold stress. Clin Auton Res 1997; 7: 93-6.

22. Fields HL, Basbaum Al. Endogenous pain control mechanisms. In: Wall PD, Melzack R, eds. Textbook of Pain. New York: Churchill Livingstone; 1984.p142-52.

23. Thoren P, Floras JS, Hoffman P, Seals DR. Endorphins and exercise: Physiological mechanisms and clinical implications. Med Sci Sports Exer 1990; 22: 417-28.

24. Casey KL. Forebrain mechanisms of nociception and pain: analysis through imaging. Proceeding of the National Academy of Sceinces USA 1999; 96:7668-74.

25. Nayak S, Shiflett SC, Eshim S, Levine FM. Culture and gender effect in pain beliefs and the prediction of pain tolerance. Cross-cultural Research 2000; 34: 135.

26. Zeltzer LK, Fanurik D, LeBaron S. The cold pressor pain paradigm in children: feasibility of an intervention model (part II). Pain 1989; 37: 305-13.

27. Marieb Elaine RN. Human Physiology and anatomy. 4th Edition. Menlo Park, CA: Benjamin/Cummings Science ;1998.

28. Clark WC and Clark S. Pain responses in Nepalese porters. Science 1980; 209: 40.

29. Iadarola MJ, Brady LS, Draisci G, Dubner R. Enhancement of dynorphin gene expression in spinal cord following experimental inflammation: stimulus specificity, behavioral parameters and opoid receptor binding. Pain 1988; 35(3): 313-26.

30. Stening K, Eriksson O, Wahren L, Berg G, Hammar M, Blomqvist A. Pain sensations to the cold pressor test in normally menstruating women: comparison with men and relation to menstrual phase and serum sex steroid levels. Am J Physiol Regul Integr Comp Physiol 2007; 293: 1711-6. 
31. Kowalczyk WJ, Evans SM, Bisaga AM, Sullivan MA, Comer SD. Sex differences and hormonal influences on response to cold pressor pain in humans. J Pain 2006; 7(3): 151-60.

32. Droste C. Physical exercise, endogenous opiates and pain regulation. Schmerz 1991; 5:138-47.

33. Ryan SM, Maier SF. The estrous cycle and estrogen modulate stressinduced analgesia. Behavioral Neuroscience 1988; 102: 371-380.

34. Al'Absi M, Wittmers LE, Ellested D, Nordehn G, Kim SW, Kirschbaum $C$ et.al. Sex differences in pain and hypothalamic-pituitary adrenocrotical responses to opioid blockade. Psychomo Med 2004; 66: 198-296.

35. Kowalczyk WJ, Evans SM, Bisaga AM, Sullivan MA, Comer SD. Sex differences and hormonal influences on response to cold pressor pain in humans. J Pain 2006; 7: 151-160.
36. Dixon KE, Thorn BE, Ward LC. An evaluation of sex differences in psychological and physiological responses to experimentally induced pain: a path analytic description. Pain 2004; 112: 188-196.

37. Edwards RR, Haythornthwaite JA, Sullivan MJ, Fillingim RB. Catastrophizing as a mediator of sex differences in pain: differential effects for daily pain versus laboratory-induced pain. Pain 2004; 111: 335-341.

38. Thorn BE, Clements KL, Ward LC, Dixon KE, Kersh BC, Boothby JL et.al. Personality factors in the explanation of sex differences in pain catastrophizing and response to experimental pain. Clin J Pain 2004; 20: $275-282$. 